\title{
An Analytical Model for the Prediction of a Micro-dosimeter Response Function
}

\author{
F. F. Badavi ${ }^{\mathrm{a}}$, M. A. Xapsos ${ }^{\mathrm{b}}$ \\ ${ }^{\text {a } C h r i s t o p h e r ~ N e w p o r t ~ U n i v e r s i t y, ~ N e w p o r t ~ N e w s, ~ V A . ~ U S A ~}$ \\ ${ }^{\mathrm{b}}$ Goddard Space Flight Center, Greenbelt, MD. USA
}

\begin{abstract}
A rapid analytical procedure for the prediction of a micro-dosimeter response function in low Earth orbit (LEO), correlated with the Space Transportation System (STS, shuttle) Tissue Equivalent Proportional Counter (TEPC) measurements is presented. The analytical model takes into consideration the energy loss straggling and chord length distribution of the detector, and is capable of predicting energy deposition fluctuations in a cylindrical micro-volume of arbitrary aspect ratio (height/diameter) by incoming ions through both direct and indirect ( $\delta$ ray) events. At any designated (ray traced) target point within the vehicle, the model accepts the differential flux spectrum of Galactic Cosmic Rays (GCR) and/or trapped protons at LEO as input. On a desktop PC, the response function of TEPC for each ion in the GCR/trapped field is computed at the average rate of 30 seconds/ion. The ionizing radiation environment at LEO is represented by O'Neill's GCR model (2004), covering charged particles in the $1 \leq Z \leq 28$ range. O'Neill's free space GCR model is coupled with the Langley Research Center (LaRC) angular dependent geomagnetic cutoff model to compute the transmission coefficient in LEO. The trapped proton environment is represented by a LaRC developed time dependent procedure which couples the AP8MIN/AP8MAX, Deep River Neutron Monitor (DRNM) and F10.7 solar radio frequency measurements. The albedo neutron environment is represented by the extrapolation of the Atmospheric Ionizing Radiation (AIR) measurements. The charged particle transport calculations correlated with STS 51 and 114 flights are accomplished by using the most recent version (2005) of the LaRC deterministic High charge (Z) and Energy TRaNsport (HZETRN) code. We present the correlations between the TEPC model predictions (response function) and TEPC measured differential/integral spectra in the lineal energy (y) domain for both GCR and trapped protons, with the conclusion that the model correctly accounts for the increase in flux at low y values where energetic ions are the primary contributor. We further discuss that, even with the incorporation of angular dependency in the cutoffs, comparison of the GCR differential/integral flux between STS 51 and 114 TEPC measured data and current calculations indicates that there still exists an underestimation by the simulations at low to mid range y values. This underestimation is partly related the exclusion of the secondary pion particle production from the current version of HZETRN.
\end{abstract}

Keywords: STS dosimetry, TEPC, HZETRN

* Corresponding author. Phone: +1 7578641410

Fax: +1 7578648094

E-mail address: francis.f.badavi@nasa.gov 


\section{Introduction}

Long duration manned space travel, outside the protective cover of the Earth's magnetosphere, to regions where there exists the possibility of serious biological injury due to energetic solar proton events (SPE) and GCR events, require an understanding of the short and long term effects of the interaction of ionizing radiation with body organs. For the short term SPE, the primary concerns are the acute effects arising from the sudden exposure to large dose levels of solar protons. In general, where feasible, the severity of an SPE dose build up in the crew quarter(s) can be sufficiently reduced with adequate augmentation of polymer based low charge number $(Z)$ materials. On the other hand, the cumulative exposure to the low intensity, high linear energy transfer (LET) components of GCR pose a serious technical challenge to the ionizing radiation protection research community. Not only is there a lack of adequate human data to analyze and assess the effects of high LET particles, but there also exist uncertainties in the knowledge of heavy ion interaction with body organs as ions penetrate through the shielding materials.

The interaction of SPE and GCR generated ions with nuclei of shielding structures (materials) and body organs result in energy degradation and nuclear fragmentation of the radiation field. The nuclear fragmentation cascade processes produce secondary and subsequent generation reaction products that alter the elemental and isotopic composition of the transported radiation field. Only with detailed knowledge of the radiation field at specified organ locations of the crew can one begin to assess the short and long term health risks due to exposure to space radiation.

A feature of ionizing radiation is its discontinuous nature of interaction with matter. That is, the deposited energy into a medium consists of discrete events, with energy partitioning among ionization and excitation processes. However, traditional quantities of biological interest such as LET, absorbed dose (D) and dose equivalent (H), are statistically averaged quantities that disregard the resulting random fluctuations of the interaction. It is therefore a general practice to assume that the energy deposited by an incident ion in a target volume is also the statistically averaged energy lost locally by the same ion within the volume. Under certain combinations of target (detector) physical size, ion type and its corresponding energy, the above assumption can be used with negligible errors (ref. 1) to show that, for instance, at a tissue site of $2 \mu \mathrm{m}$, depending on the ion type, the energy loss straggling becomes important only if the energy of incident ions exceeds the range of $5-20 \mathrm{~A}-\mathrm{MeV}$. From a computational simulation point of view, the above assumption eliminates the need to resort to Monte Carlo (MC) simulation of interactions, which may require the inclusion of electron (delta ray) transport. Furthermore, because of the broad range of particle charge and energy in the GCR spectra, any transport computation of such spectra through a target material, using a statistical approach, would be a very time consuming computational task. It must, however, be stated that as the target (detector) size decreases down to fractional micrometer $(\mu \mathrm{m})$ or nanometer $(\mathrm{nm})$ domain, the energy deposited in the site fluctuates and can differ significantly from the energy loss (LET) of the interacting ion. Indeed, it was because of the difficulties encountered in interpreting measured LET results in small sites that the randomness of the deposited events was eventually understood, with the subsequent realization that LET itself was possibly less important than the raw data, which represented the actual deposited energy spectra. This conclusion, by different microdosimetric groups, led to the suggestion that the usual LET dependent quality factor $(\mathrm{Q})$ be replaced by a lineal energy $(y)$ dependent $Q$ for use in radiation protection studies.

Due to the practical limitation in estimating the y spectra in a small site, in the field of computational shielding design, the implementation of $y$ dependent $\mathrm{Q}$ has turned out to be a challenging task. However, the use of $\mathrm{y}$ as a microdosimetric tool, to understand the behavior of the spectral distribution of radiation components of different LET, has led to the general conclusion that LET is only one of many factors that determines the extent of energy deposition in a micro-volume, with other contributing factors being ion range, energy loss straggling and energy dissipation by secondary electrons. Indeed, various studies have shown that there is only a narrow region for which LET and y can be approximated as equal to each other.

Since the estimation of the health risk to the crew from space radiation can be based on the knowledge of $\mathrm{H}$ derived from LET, measurements of high LET spectra have been carried out since the Gemini flights (ref. 2). The usual method of obtaining LET is based on passive plastic track detectors with limited LET range, such as; nuclear emulsion, CR 39 and Lexan. With a typical lower bound LET threshold of $5 \mathrm{keV} / \mu \mathrm{m}$, these detectors cannot detect electrons, and their efficiency for detection of secondaries, such as pions or kaons, are not well established. They also experience detection-resolution limitations above LETs of $250-300 \mathrm{keV} / \mu \mathrm{m}$, where the track length is very short, and hence analyzing the track becomes a challenging task. Finally, because of the passive nature of these detectors, the separation of GCR from trapped particles for LEO flights is difficult. 
In contrast to the limitations of passive detectors, TEPC detectors simulate a small tissue site, and can provide time resolved dose and y spectra. The TEPC used in the STS consists of a cylindrical detector, $1.78 \mathrm{~cm}$ in height and diameter, simulating a $2 \mu \mathrm{m}$ tissue site that is bounded by tissue equivalent plastic. The instrument covers a y range of $0.4-1250 \mathrm{keV} / \mu \mathrm{m}$. The energy resolution of the electronics is $0.1 \mathrm{keV} / \mu \mathrm{m}$ below $20 \mathrm{keV} / \mu \mathrm{m}$, and $5 \mathrm{keV} / \mu \mathrm{m}$ above $20 \mathrm{keV} / \mu \mathrm{m}$. A complete description of the instrument can be found in the work of Badhwar et al. (ref. 3 ).

In the past, MC simulations have traditionally been the method of choice to model energy deposition by ions in a microvolume. Although results from such simulations have proven to be valuable, they generally involve the implementation of sophisticated computer codes and time consuming scoring techniques, requiring large quantities of input information, and often require the tedious task of how to interpret the results. For a complex radiation field, such as GCR, with broad energy spectra spanning many orders of magnitude and ion composition covering essentially the entire periodic table, utilizing any MC methodology is a time-consuming approach, as it is very difficult to use any MC method to cover all the species and energy ranges of GCR spectra.

In contrast to $\mathrm{MC}$ simulations of the past, recently a number of analytical descriptions for representing the stochastic energy deposition and ionization produced by energetic ions passing through absorber sites of submicron dimension have been developed (refs. 4, 5). Xapsos (refs. 6,7) developed an analytical approach for the description of energy deposition and ionization due to single and multiple events (ionization due to combined effects of multiple ion tracks), that can be used for any GCR, SPE and trapped proton spectrum, with arbitrary energy and micron-size site diameter, with simple inputs of physical quantities. The approach of references 6 and 7 for single event distributions is used herein to obtain the response of the STS TEPC due to incident GCR ions and trapped protons, as an attempt to provide accurate prediction for comparison with STS measurements. In this paper, the analytical approach to compute the altered radiation level and energy deposition spectrum of each ion species, is to couple the model of references 6 and 7 with the computationally efficient High charge (Z) and Energy TRaNsport (HZETRN) code, which provides a radiation analysis tool suitable for the study of space mission shielding design (refs. 8 - 10).

The first step in the computational process begins with the establishment of an appropriate environmental model. For the LEO environment as applied to a pressurized vehicle, the most important contributors to the deposition of ionizing radiation energy are the GCR and trapped protons. Here, the paper briefly introduces the GCR component of the LEO radiation field and directional dependent geomagnetic transmission due to GCR. It then briefly describes the albedo neutron spectrum as the result of the interaction of GCR with Earth's atmosphere. Next, the highly directional (vectorial) nature of the proton flux, which roughly constitutes half of the total cumulative exposure for long duration missions, is briefly described, noting that the instantaneous trapped protons dose rates are much higher during the approximately 5 to 10 minutes of South Atlantic Anomaly (SAA) transits. During the transits, both omni-directional and vector proton flux vary from near zero to maximum values, and directionality is controlled by the vehicle orientation with respect to the magnetic field vector components. Consequently, an added degree of complexity is introduced with the time variation of proton flux spectra along the orbit, for which individual transport properties through the shield medium must be taken into account. With the external radiation environment defined, the paper then briefly describes the deterministic high energy heavy ion transport code HZETRN, developed at NASA Langley Research Center (LaRC), to describe the attenuation and interaction of the LEO environment particles and to calculate dosimetric quantities of interest. This is followed by the description of the energy deposition model in terms of $y$, and how LET related quantities are defined. Finally, the two STS geometries defined by the Computer Aided Design (CAD) models, representing the location of TEPC detectors, are used to calculate the differential and integral response function y and LET spectra, and are compared with STS 51 and 114 TEPC measurements. The paper is then concluded by discussing the limitations of the developed TEPC response function as used in this study.

\section{LEO Environment and Transport Models}

The LEO environment consists of three main sources, GCR that penetrate the geomagnetic field, albedo neutrons from GCR interaction with the Earth's upper atmosphere, and particles trapped in the geomagnetic field. The primary limitations in the traditionally used environmental models are that the trapped proton models AP8MIN/AP8MAX for solar minimum and maximum are time and direction independent, and that the vertical geomagnetic cutoff is used to describe the transmitted GCR at LEO. Improvements to these traditional LEO environmental models, by introducing a dynamic and anisotropic trapped protons environment and general geomagnetic cutoff model, are briefly described next. 


\subsection{GCR Environment}

Models of free space GCR environment (refs. 11-13) developed in the past two decades have provided the most realistic description of the interaction of incoming GCR from outside the heliosphere with solar activity. The model of reference 11 and its updated version by O'Neill (ref. 14) is currently used as GCR input to HZETRN. This model is based on fitting the existing balloon and satellite measured energy spectra from 1954 - 1992 and more recent measurements from Advanced Composition Explorer (ACE) satellite from 1997 - 2002, to the stationary Fokker-Planck equation to solve the diffusion, convection and energy loss boundary value problem, and obtain an estimate of the appropriate diffusion coefficient. In addition, correlation of the diffusion coefficient to the Climax neutron monitor data, which exhibits an odd - even cycle with a 22 year period, enables the estimation of the diffusion coefficient at times that direct observational data are not available. The latest implementation of this model, (2004), accurately accounts for the solar modulation of hydrogen through nickel ( $\mathrm{H}$ - Ni), by propagating the local interplanetary spectrum (LIS) of each element through the heliosphere. The model provides a single value of the deceleration parameter, $\Phi(t)$, describing the level of solar cycle modulation, and determines the GCR differential energy spectrum for elements $1 \leq Z \leq 28$ at a given radial distance from the sun.

\subsection{Geomagnetic Transmission Factor}

In the past, the commonly used geomagnetic transmission factor was based on the extrapolation of a world map of vertical cutoff rigidities by Smart and Shea as described in reference 15. In this model, it was assumed that there is no transmission below the vertical cutoff, and 100\% transmission (excluding the Earth's shadow) above the vertical cutoff; in fact, there is partial transmission, dependent on the angle of incidence relative to the east direction. It is convenient to characterize the geomagnetic interaction of GCR particles in terms of rigidity, $\mathrm{R}$ (momentum/unit charge), rather than energy. A common method of representing GCR transmission through the geomagnetic field is use of a computed local vertical cutoff rigidity, $R_{V C}$, for which transmission is unity for $R>R_{V C}$ and zero otherwise. This simple dipole approximation may be improved upon by utilizing detailed calculations of vertical cutoff rigidity evaluated from the multipole field models. Global maps of cutoff rigidity are available, and have been incorporated in the present work from reference 15 . The temporal variation of GCR flux is also taken from the detailed vertical cutoff calculations for the time intervals covering most of the last half century, and reflecting the varying field strength observed during this period. In the present model, we use the International Geomagnetic Reference Field (IGRF) model evaluated for arbitrary dates from 1945 to 2020 (ref. 15).

\subsection{Albedo Neutron Environment}

Albedo neutrons result from the interaction of GCR with the Earth's atmosphere. As the GCR intensities are modulated by solar activity, so are the atmospheric neutrons modulated with time. The atmospheric neutron model is a parametric fit to data gathered by LaRC studies of radiations at Supersonic Transport (SST) altitudes in the years 1965-1971, covering the rise and decline of solar cycle 20 (1965-1977). Scaling of the data with respect to geomagnetic cutoff, altitude, and modulation of the Deep River Neutron Monitor (DRNM) was found to allow mapping of the environment to all locations at all times, resulting in an empirically based model for atmospheric neutrons. In this model, as described in reference 15, the leakage flux $F_{L}(E)$ is closely related to the azimuthal differential flux $\phi(E, \Omega)$ at the top of the atmosphere as

$$
F_{L}(E)=\int \phi(E, \Omega) \cos \theta d \Omega
$$

where $E$ is the kinetic energy and $\cos \theta$ is the direction $\operatorname{cosine}$ of the velocity vector with the zenith (i.e., $\phi(E, \Omega)=0$ for $\cos \theta$ $<0$ ). Parameterization of the numerical result of equation 1 , produces an energy dependent fit to the leakage flux $F_{L}(E)$ in the form of

$$
\begin{array}{ll}
F_{L}(E)=0.065 / E & E \leq 10 \mathrm{MeV} \\
F_{L}(E)=0.0026 \exp (-0.011 E) & E>10 \mathrm{MeV}
\end{array}
$$

In this paper, the amplitude of leakage flux $F_{L}(E)$ at the top of the atmosphere, as fitted by equation 2, is extrapolated to any LEO altitude using $\mathrm{r}^{-2}$ neutron attenuation factor, where $\mathrm{r}$ is the atmospheric height in $\mathrm{g} / \mathrm{cm}^{2}$. 


\subsection{Trapped Proton Environment}

The commonly accepted trapped proton environment relies on the assumption that trapped particles are isotropic, resulting from the omni-directional fluence description, and the use of the vertical geomagnetic cutoff to describe the transmitted GCR. These models have been relatively successful in describing the radiation environment aboard the highly maneuverable STS where anisotropies tend to be averaged (smeared) out. This averaging process is due to the spinning and random STS orientations which wash out protons anisotropies, and hence directionality in the trapped protons flux is generally ignored for STS flights, with the omni-directional flux being used for STS dosimetric calculations.

The particles trapped in the geomagnetic field were modeled from data obtained during two epochs of solar cycle 20 (solar minimum of 1965 and solar maximum of 1970), and are used with the geomagnetic fields on which the Mcllwain integral adiabatic invariant B/L maps were prepared. The 1965 analysis using the magnetic field model of Jensen and Cain (ref. 15) resulted in the particle population maps AP8MIN, and the 1970 analysis using the magnetic field model of Goddard Space Flight Center (GSFC) 12/66 extended to 1970 resulted in the particle population maps of AP8MAX (ref. 15).

Practically all of the trapped protons flux in LEO $(\sim 300-\sim 1000 \mathrm{~km})$ are encountered in the SAA region. The flux of trapped protons exhibits pronounced directional characteristics, since this is a region close to a "mirror point" where the protons pitch angle with respect to the magnetic field vector is close to $90^{\circ}$. Within the SAA, trapped protons attain their minimum mirror point altitudes, displaying planar geometry as their dominant feature.

Due to orbital precession, STS and International Space Station (ISS), during their 5 to 10 minutes passage through the SAA, encounter trapped protons from both ascending and descending node directions. Because the radiation incident on the outer surface of the spacecraft is required for shield evaluation, and attitude of spacecraft is never fixed, but has limited cycles due to required re-orientation maneuvers, angular distribution averaged over spacecraft attitude in the region of radiation encounter needs to be evaluated. This is accomplished by relating the orientation in the spacecraft frame through yaw, pitch, and roll to the local vertical reference frame where the radiation environment is evaluated. In this work, 970 ray directions are used to evaluate the boundary conditions for shield evaluation.

\subsection{Charged Particles Transport Model}

The propagation of GCR ions and their secondary byproducts through matter (shield) is described by the Boltzmann equation. Wilson et al. (refs. 8-10) provided a numerical solution to this equation using the straight ahead and continuous slowing down (CSDA) approximations. These approximations, which result in negligible error for heavy ions in space applications, offered a very efficient engineering algorithm for large scale mission studies for which statistical methodologies, such as Monte Carlo (MC) would have been unacceptably time consuming to produce results. Several engineering solutions obtained using HZETRN, in conjunction with a ray-tracing technique and a CAD model for complex 3D geometries and material compositions, have previously demonstrated the computational efficiency of HZETRN (ref. 16), albeit LEO based studies involving STS geometry conducted so far are still limited to the available geometry package that assumes a single aluminum equivalent material, and further assumes an isotropic distribution of the incident radiation field. As the need to improve HZETRN for usage in space mission design studies was recognized, further improvements were made, which included the augmentation of fully energy dependent interaction cross sections, and expanded isotopic composition for the fragmented secondaries. In addition, considerable improvements in the nuclear database through comparison with laboratory experiments using accelerator and space flight measured data were made (refs. 17-19).

\section{Description of Two Components Analytical Model}

As an ion traverses randomly through a detector volume of micron size, the amount of ionization in the volume depends on a number of factors including the actual path length of the ion in the volume, the energy transported by the electrons out of the volume and the energy partitioning between ionization and inelastic excitations. In addition, ions which do not traverse the volume but pass by within proximity of the target may also deposit some of their energy by injecting electrons into the volume. For a micro-volume target size, the process of energy deposition by an incident ion is depicted in figure 1 where the solid line denotes ion passage through or near the site, and the dotted line denotes transport of energy away from the ion track by secondary electrons. 


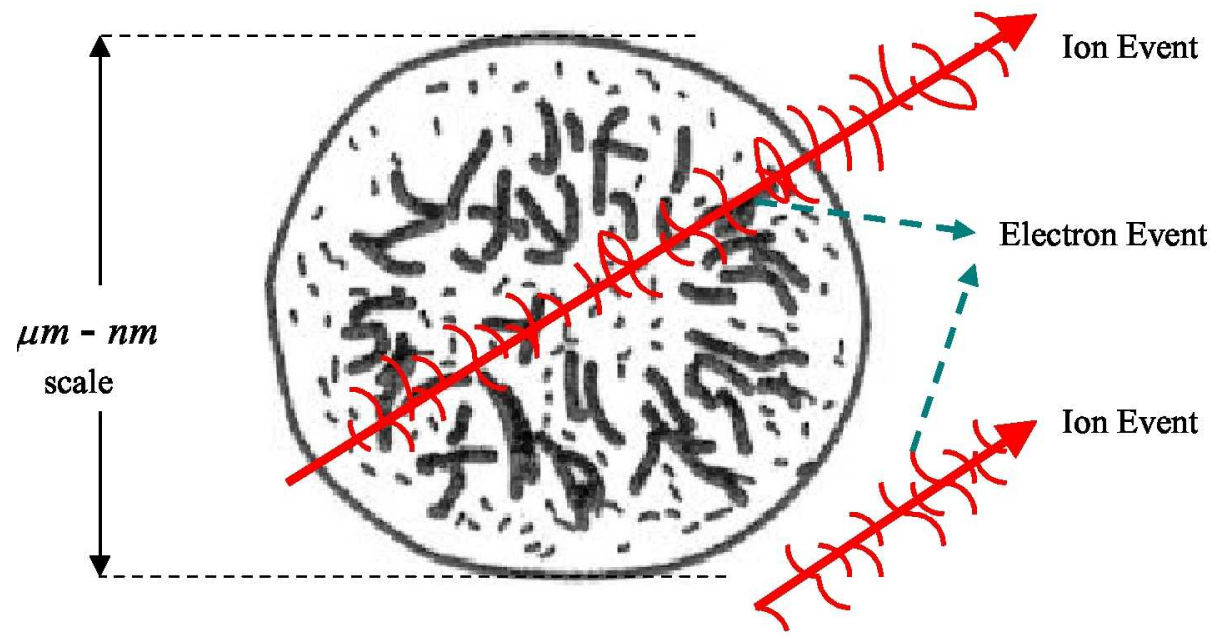

Figure 1. Schematic of ion and electron energy deposition processes within a micro-volume.

In this work, the analytical approach, developed in references 6 and 7 to obtain a solution for the ionization spectrum produced in a small volume by the passage of ions and secondary electrons, is used to develop a TEPC response function for the analysis of STS measured data. Provided here is a brief description of the analytical approach and its extension to evaluate TEPC response due to ionizing space radiation.

\subsection{Modeling of Ion Events Distribution}

With the assumption that the traversing ion loses only a small fraction of its energy as it travels through the target medium, the average energy deposited in the micro-volume is given by (ref. 7)

$$
\bar{E}_{\text {ion }}=f_{\text {ion }} L_{i o n} s_{i o n}
$$

where $L_{i o n}$ is the linear energy transfer (LET) of the traversing ion; $S_{i o n}$, the path length through the target; and $f_{i o n}$, the fraction of the energy initially deposited which remains within the site; that is, the fraction not carried out of the site as kinetic energy of the secondary electrons. For a given ion, $f$ can be expressed as (ref. 6 )

$$
f_{i o n}=\frac{\ln \left[\left.\frac{E_{e, \max }\left(\Delta+\Delta_{1}+\Delta_{2}\right)}{I^{2}}\right|^{2}\right.}{2 \ln \left(\frac{E_{e, \max }}{I}\right)}, \Delta \leq E_{e, \max }
$$

where $E_{e, \max }$ is the maximum kinetic energy of an electron which results from a collision with the incident ion, and in units of $\mathrm{keV}$ is defined as

$$
E_{e, \max }=2.179 \frac{E_{\text {ion }}}{m_{\text {ion }}}=2.179 e_{\text {ion }}
$$

with $m_{i o n}$ as the mass of the ion, $E_{i o n}$ as the kinetic energy of the incident ion, and $e_{i o n}$ as the kinetic energy of the incident ion per amu. $\Delta$ is the cut-off energy, which is determined by the dimensions of the sensitive volume, and $I$ is the mean excitation energy of the target medium.

In terms of $E_{e, \max }, \Delta$ and $I$, quantities $\Delta_{1}$ and $\Delta_{2}$ in equation 4 are defined as

$$
\Delta_{1}=\Delta\left(1-\frac{\Delta}{E_{e, \max }}\right)
$$

and

$$
\Delta_{2}=I\left(1-\frac{\Delta}{E_{e \cdot \max }}\right)
$$


In equation $6, \Delta_{1}$ represents the energy transfer in the micro-volume by secondary electrons produced within the volume, which subsequently escape from it, and $\Delta_{2}$ represents the energy of ionization and excitation contained in atoms in the microvolume that experience the primary interaction when produced secondary electrons escape the volume.

The average number of electron-hole pairs produced in the target as the ion traverses a path length of $S_{\text {ion }}$ is defined as

$$
x_{i o n} \equiv \frac{\vec{E}_{i o n}}{W_{i o n}}
$$

where $W_{i o n}$ is the average energy required to produce an electron-hole pair.

As the detector (target) size reaches micron dimension, the fluctuation of energy deposition processes become increasingly important. The relative variance of deposited energy for an ionization event $V_{i o n}$ is given by (ref. 20)

$$
V_{i o n}=V_{s t r, i o n}+V_{F, i o n}
$$

where $V_{s t r, i o n}$ is relative variance of energy loss straggling and $V_{F, \text { ion }}$ is relative variance of the Fano fluctuations (ref. 21), as related to the energy partitioning. The latter contribution is included if ionization is the process of concern, as in the case of TEPC, and is omitted if energy deposition is the process of concern.

Equations (3), (7) and (8) indicate that the probability distribution function for ionization produced by the random traversal of an ion through the volume requires knowledge of path length distribution and energy loss straggling including Fano fluctuations. The probability distribution function for the ion's path length can be obtained from the chord length distribution of the detector (target) volume under the assumption that the ion is energetic enough to travel in straight lines through the volume. In this process, the energy loss straggling can be approximated by a lognormal distribution with all the required parameters given in terms of relative variance of the random variables involved in the energy deposition process. This is so because with each collision, the ion loses some random fraction of its energy that is proportional to its energy before the collision. Given $p_{i o n}\left(x_{i o n}, s_{i o n}\right)$ as the probability density distribution function for the lognormal process to produce $x_{i o n}$ ionizations related to the path length $s_{i o n}$ of an incident ion, the overall probability density distribution can be expressed as

$$
F_{i o n}\left(x_{i o n}\right)=\int p_{i o n}\left(x_{i o n}, s_{i o n}\right) c\left(s_{i o n}\right) d s_{i o n}
$$

where $c\left(s_{i o n}\right)$ is the normalized chord length density distribution function of the target micro-volume. The quantity $F_{i o n}\left(x_{i o n}\right)$ is the normalized probability density that a single ion produces $x_{i o n}$ electron-hole pairs within the restricted target volume upon crossing the site.

The lognormal distribution is represented as

$$
p_{i o n}\left(x_{i o n}, s_{i o n}\right)=1 /\left(\sqrt{2 \pi} \sigma_{i o n} x_{i o n}\right) \exp \left[-\left(\ln x_{i o n}-\mu_{i o n}\right)^{2} / 2 \sigma_{i o n}^{2}\right]
$$

and the parameters of the lognormal distribution are related to the mean and relative variance of the number of ionizations according to (ref. 22)

and

$$
\mu_{i o n}=\ln \left(x_{i o n}\right)-0.5 \sigma_{i o n}^{2}
$$

$$
\sigma_{i o n}^{2}=\ln \left(1+V_{i o n}\right)
$$

where all variables are a function of path length $s_{i o n}$.

Note that equation (10) represents the probability distribution of any random variable whose logarithm is normally distributed. Specifically, if $x$ is a random variable with a normal distribution, then $\exp (x)$ has a lognormal distribution profile. A variable can be considered to have a lognormal distribution if it is made of the multiplicative product of a series of small independent factors, and it is only due to the discontinuous nature of radiation interaction with matter that the proper distribution of interaction in the micro-volume can be represented by a lognormal distribution. Figure 2 is the representation of the probability density function (PDF) (left) and cumulative distribution function (CDF) (right) of a lognormal distribution with a mean of $\mu=0$ and standard deviation $\sigma$ in the range of $1 / 8-4$. 

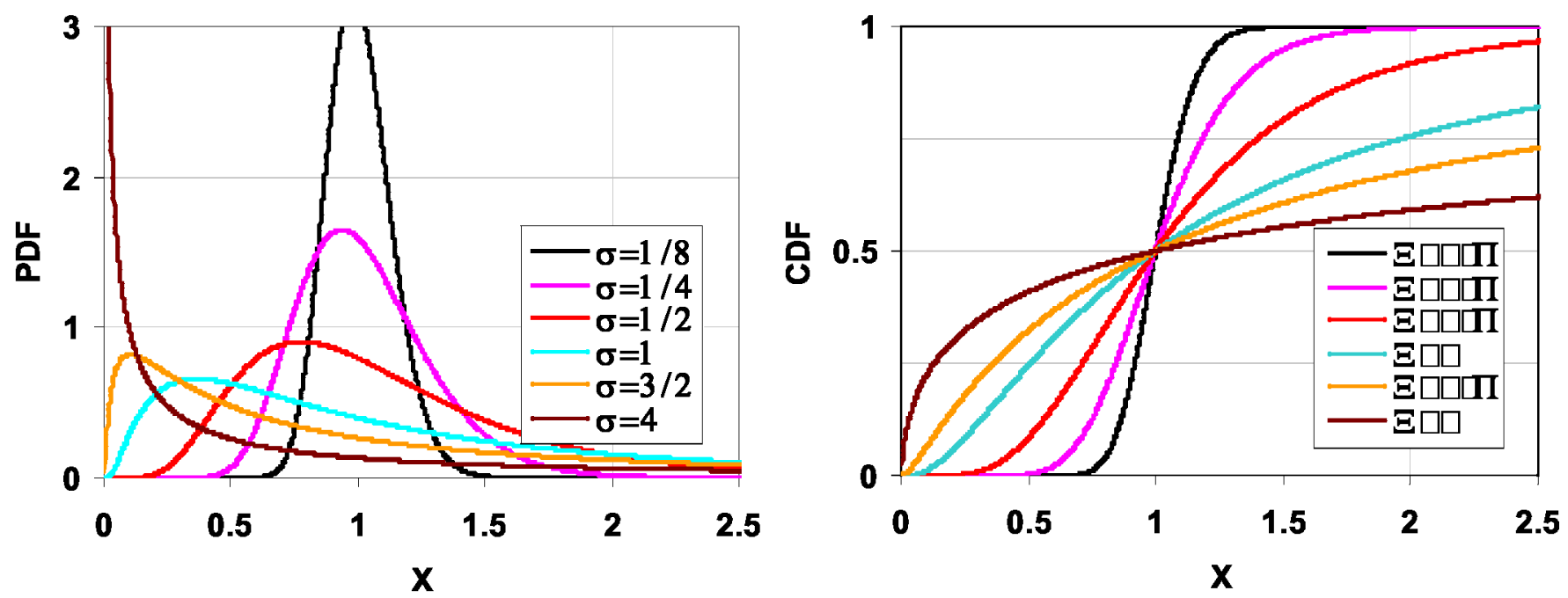

Figure 2. Lognormal distribution PDF (left) and CDF (right) for $\mu=0$ and $1 / 8 \leq \sigma \leq 4$.

The derived formulation for the relative variance depended only on easily obtainable macroscopic quantities such as LET and range of the ion, and the evaluation of equation (10) does not rely on curve fitting from existing MC results. In addition, parameters $\mu_{i o n}$ and $\sigma_{i o n}$ needed for calculating energy loss straggling simply depend on the detector composition and size, particle types and energy values. The approach here is to obtain an analytical expression for the relative variance $V_{i o n}$ so that equation (9) is readily solvable for any given size of detector for the randomly incident ions observed in space.

The relative variance of energy loss straggling for an ion can be defined as

$$
V_{\text {str }, \text { ion }}=\delta_{2} / E_{\text {ion }}
$$

where $\delta_{2}$ is the energy weighted mean of the energy deposited per ion-electron collision in the site (ref. 7). For a micron sized volume of tissue traversed by an ion with energy greater than $3 \mathrm{~A}-\mathrm{MeV}$ or so, the track width will be large enough to allow some of its deposited energy to be carried away from the volume by the electrons. The fraction of such energy loss is treated analytically (refs. 23, 24) and included in the evaluation of $E_{\text {ion }}$ (equation 4). An approximate form for $\delta_{2}$ is given by (ref. 25)

$$
\delta_{2}=A \Delta^{B}
$$

where $A$ and $B$ are material dependent constants.

Finally, the evaluation of relative variance of the Fano fluctuation is also possible through the relation (ref. 20)

$$
V_{F, \text { ion }}=F W / E_{\text {ion }}
$$

where $F$ is the Fano factor (ref. 21) and $W$ is the average energy required to produce an ion pair by the incident radiation. Values for $W$ in various media are readily available in the literature.

The required TEPC response function for space application is then obtained by extending equation (10) to all GCR ion types and energies. Assuming $\phi_{i o n}\left(s_{i o n}\right)$ is the resultant differential flux from transport calculation at the detector site due to random passage of an ion, the ionization spectrum produced at the detector from all GCR particles is given by

$$
\Phi\left(x_{i o n}\right)=\sum_{i o n} \int \phi_{i o n}\left(s_{i o n}\right) F\left(x_{i o n}\right) d s_{i o n}
$$

Note that $\Phi\left(x_{i o n}\right)$ can be converted to a lineal energy differential spectrum $\psi\left(y_{i o n}\right)$ through the relation $y_{i o n}=x_{i o n} W / \bar{c}_{i o n}$, where $\bar{c}_{i o n}$ is the average chord length and $y_{i o n}$ is lineal energy. 


\subsection{Modeling of Electron Events Distribution}

In the case where the ion misses the target volume, there still is a probability that energy can be deposited in the site by an indirect ionic event through electron deposition. In comparison with ion events, dealing with electron events is generally more complicated due to its energy distribution within the irradiated volume. The average energy $\bar{E}_{e}$ and average number of electron-hole pairs $\bar{x}_{e}$ deposited in the target volume by an electron traveling a distance $s_{e}$ are given by

$$
\begin{gathered}
\vec{E}_{e}=L_{e} s_{e} \\
x_{e}=\frac{\overparen{E}_{e}}{W_{e}}
\end{gathered}
$$

where $L_{e}$ is the average, slowed electron LET and is obtained by assuming a $L_{e}^{-1}$ slowing-down and $E^{-2}$ spectrum profile with $E$ being the electron energy initially produced by the incident ion (ref. 6), and $W_{e}$ is the average energy required to produce an electron-hole pair. As in the case of an ion, the path length dependent relative variance of ionization for electron events is given by

$$
V_{e}=V_{L, e}+V_{s t r, e}+V_{F, e}
$$

where $V_{L, e}$ is relative variance of the LET distribution, $V_{s t r, e}$ is relative variance of energy-loss straggling and $V_{F, e}$ is relative variance of Fano fluctuations.

\subsection{Modeling of Combined Ion and Electron Events Distribution}

What is left is to find a way to combine the normalized probability densities of ion events $f_{i o n}(x)$ with electron events $f_{e}(x)$,with $x$ being the number of electron-hole pairs produced, without distinguishing the events. The combined ionization distribution $f(x)$ is given by (ref. 6)

$$
f(x)=P f_{\text {ion }}\left(x_{i o n}\right)+(1-P) f_{e}\left(x_{e}\right)
$$

where $P$ is the fraction of ion events, and the fraction of electron events is given by (ref. 6 )

$$
1-P=\frac{\left(1-F_{i o n}\right) \bar{x}}{\bar{x}_{e}}
$$

where $\bar{x}$ is the average number of ionizations of combined ion and electron distributions. Further, $\bar{x}$ can be expressed in terms of known quantities as

$$
\frac{1}{\bar{x}}=\frac{F_{i o n}}{\bar{x}_{i o n}}+\frac{1-F_{i o n}}{\bar{x}_{e}}
$$

where $\bar{x}_{i o n}$ and $\bar{x}_{e}$ are calculated for the average path lengths $\bar{s}_{i o n}$ and $\bar{s}_{e}$. In this paper, equation (18) will be used to compute the STS TEPC response function.

\section{STS Results and Discussion}

Using the GCR and LEO environments described in section 2, HZETRN was used to generate the differential and integral y and LET spectra for STS 51 and 114. Of these, STS 51 represents a low inclination $\left(28.5^{\circ}\right)$, and STS 114 a high inclination $\left(51.6^{\circ}\right)$. Table 1 provides detailed flight information for the two flights.

\begin{tabular}{|c||c||c|}
\hline Flight year & 1993 & 2005 \\
\hline Flight designation & STS -51 & STS -114 \\
\hline Launch date & $9 / 12 / 1993$ & $7 / 25 / 2005$ \\
\hline Landing date & $9 / 22 / 1993$ & $8 / 9 / 2005$ \\
\hline Duration (days) & 9.8 & 14 \\
\hline Altitude (km) & 296 & 350 \\
\hline Inclination (deg) & 28.5 & 51.6 \\
\hline TEPC location & PB\#2 & dloc2 \\
\hline
\end{tabular}

Table 1. Various STS flight information. 
Figure 3 is a typical plot of measured dose rate versus elapsed minute by minute mission time for a portion of the STS 114 mission. The spikes represent trapped proton measurements during sequential crossings (descend-ascend) through the SAA, and the small amplitude ripples are the GCR measurements. Note that GCR minima occur near magnetic equator crossings while maxima indicate closest proximity to the magnetic poles. Due to large differences in the magnitude of trapped proton versus GCR readings, a clear separation between the two components can easily be made.

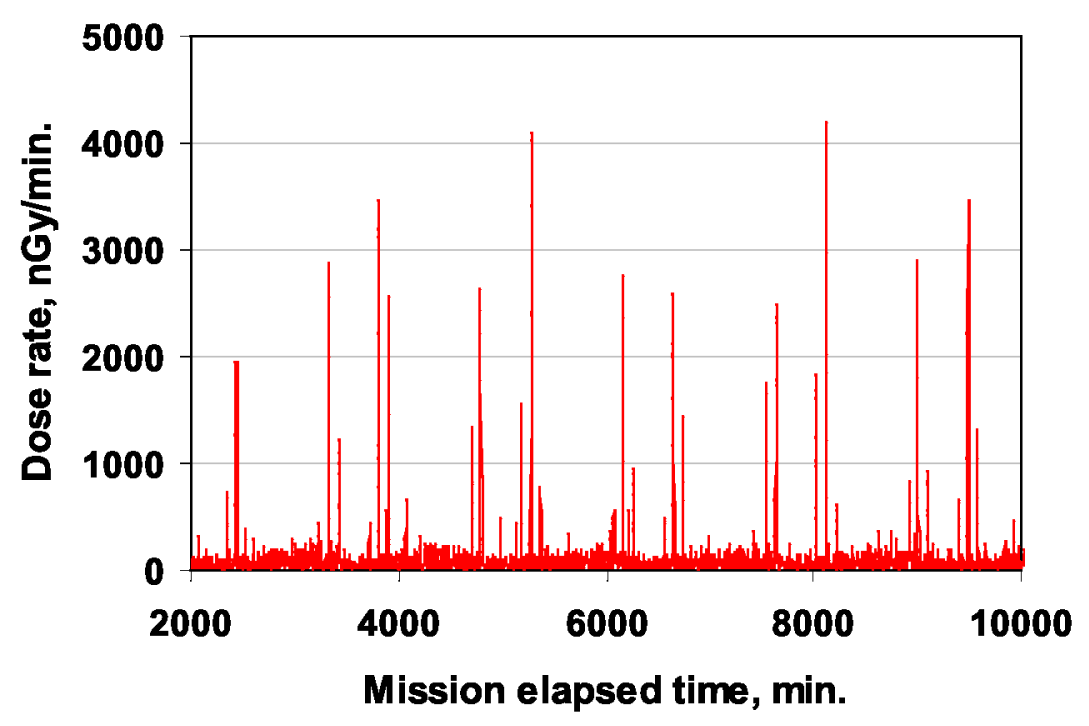

Figure 3. Dose rate versus elapsed time for STS 114 mission.

Figure 4 provides the temporal relationship between the two STS flights and the solar activity in terms of sun spot number (SSN). Flight 51 took place during the very active cycle 22 with SSN exceeding 200 during peak solar activity, while flight 114 took place during the relatively calm cycle 23 .

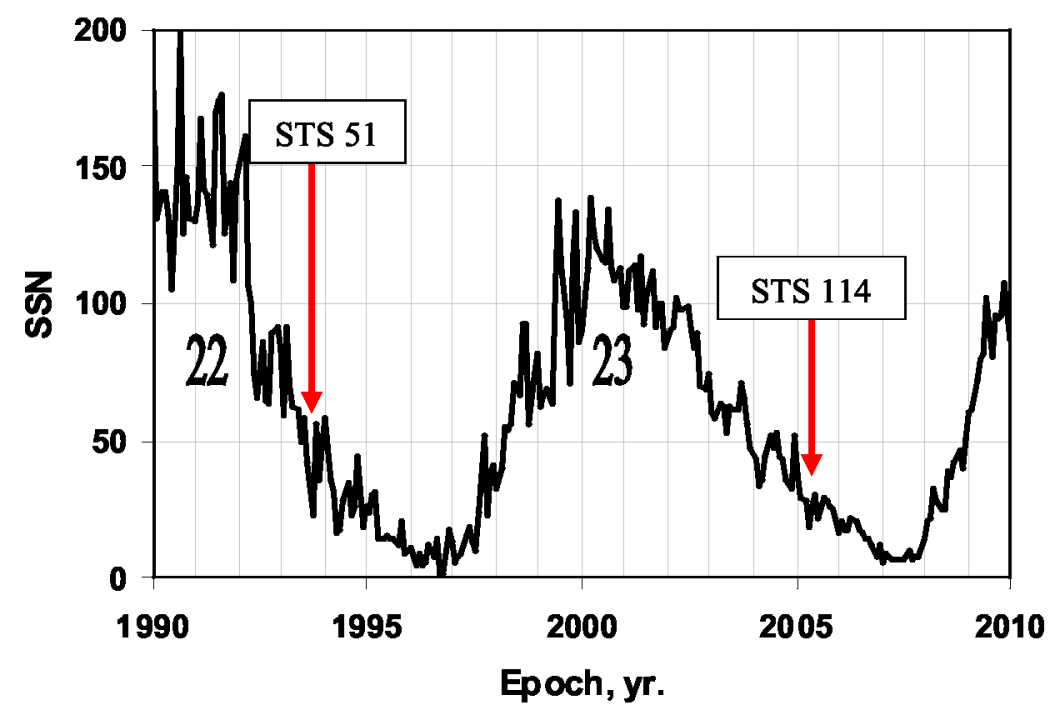

Figure 4. Temporal relationships between STS flights and SSN.

Figure 5 provides the cumulative thickness distribution for the two TEPC locations. Note that in the figure, the median ( 50 percentile) is around $2 \mathrm{~g} / \mathrm{cm}^{2}$ for the payload bay \#2 (pb\#2) and $4 \mathrm{~g} / \mathrm{cm}^{2}$ for the detector location \#2 (dloc2). This is different from the commonly stated average (mean) thickness of $11.77 \mathrm{~g} / \mathrm{cm}^{2}$ of equivalent aluminum for $\mathrm{pb} \# 2$, and 16.46 $\mathrm{g} / \mathrm{cm}^{2}$ of equivalent aluminum for dloc2, indicating that dloc2 has an additional $5 \mathrm{~g} / \mathrm{cm}^{2}$ of equivalent aluminum shielding. 


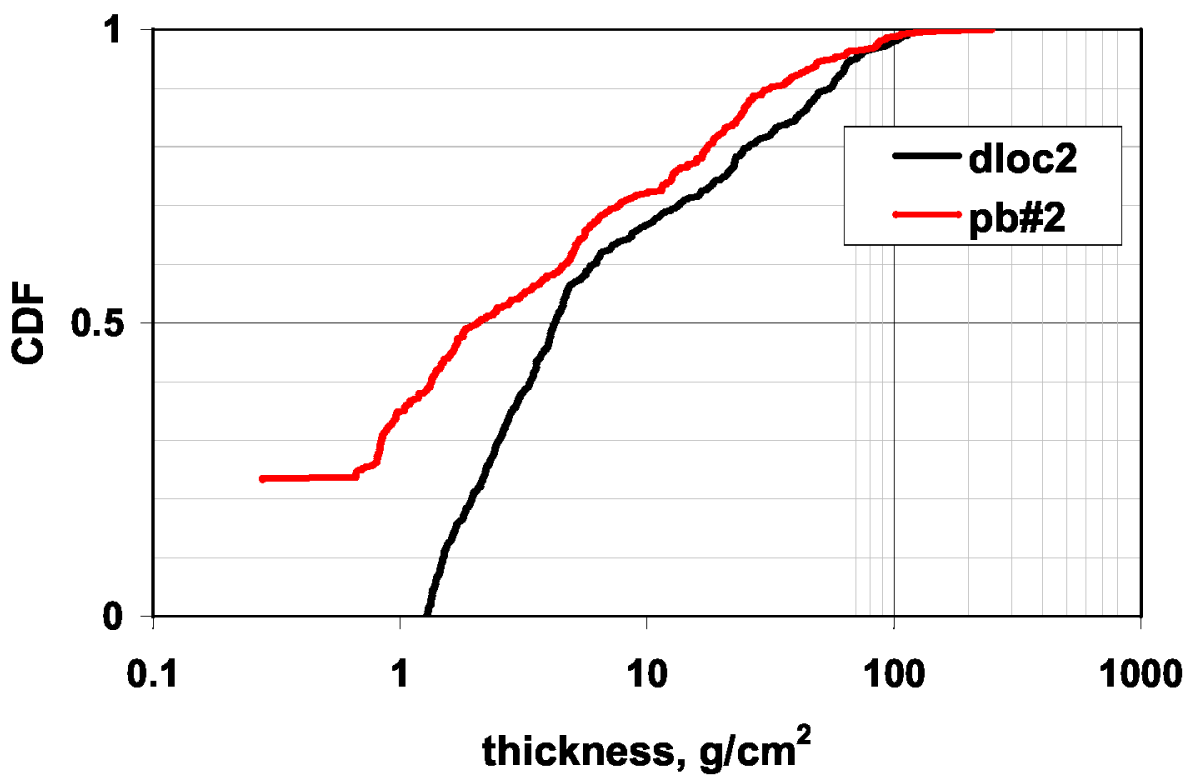

Figure 5. Cumulative Distribution Function for TEPC locations.

Assuming an isotropic incident flux of fully penetrating ions, figure 6 is the differential probability chord length distribution for a generic cylindrical detector with various height to diameter $(\mathrm{h} / \mathrm{d})$ aspect ratios. The distribution in the figure is quite sharp and peaks at 1 diameter, indicating that in order to maximize the number of ions that fully penetrate the volume of the detector, an aspect ratio of unity $(\mathrm{h} / \mathrm{d}=1)$ is desirable. The general effect of chord length variation is to smooth out the sharp peaks in the differential LET spectra due to the presence of individual ions.

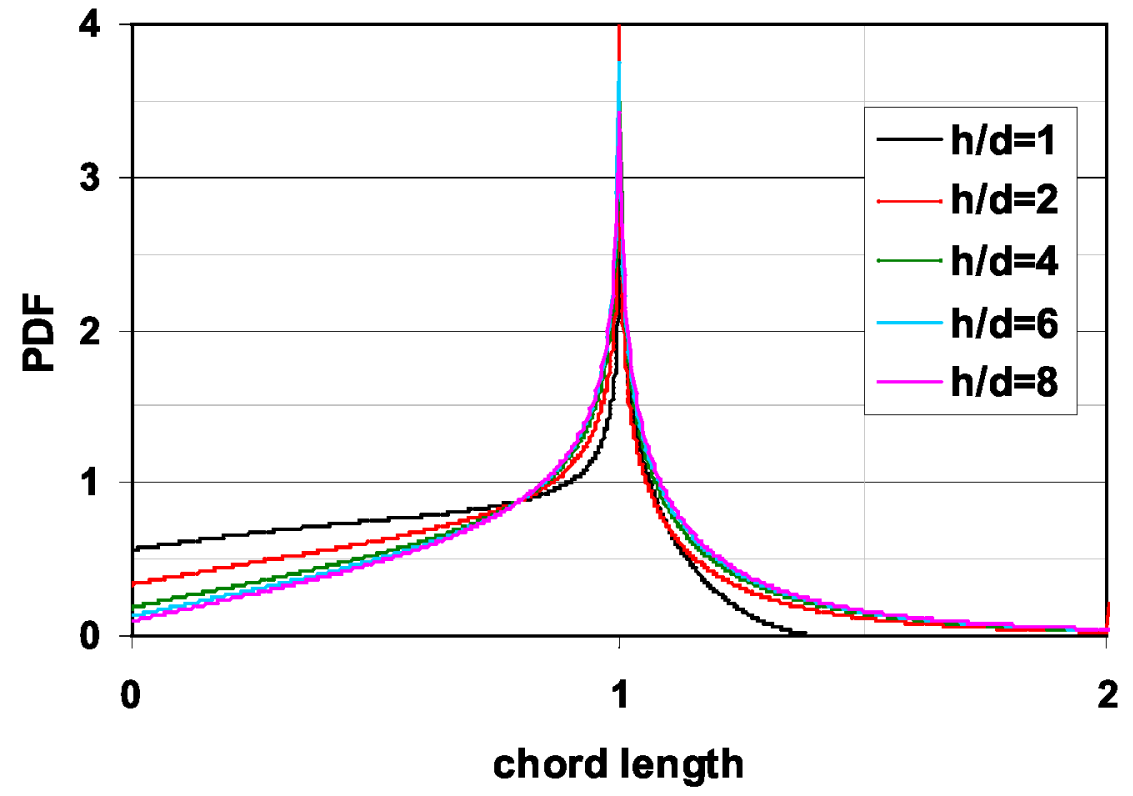

Figure 6. Differential chord distribution for ions that fully penetrate the detector volume. 


\subsection{GCR Results}

Figures $7 \mathrm{a}$ and $7 \mathrm{~b}$ are the GCR measured differential and integral y spectra for the two STS flights. The number of events detected by the instrument was converted to flux by dividing by area, solid angle and time. The projected area for the isotropically incident particles on the TEPC was computed to be $3.724 \mathrm{~cm}^{2}$, the solid angle was $4 \pi$, and time was the total time the instrument acquired data in days (ref. 3). Note that for STS 51 TEPC, there is a leakage (saturation) at minimum y value which was corrected for the later STS flights. Both figures indicate that at lower inclination (STS 51), the presence of stronger geomagnetic cut-off limits the number of ions entering the TEPC volume. Finally, the LET of minimum ionizing iron in water is about $138 \mathrm{keV} / \mu \mathrm{m}$, and figure $7 \mathrm{~b}$ indicates that the slope of the spectra above and below this value are noticeably different.

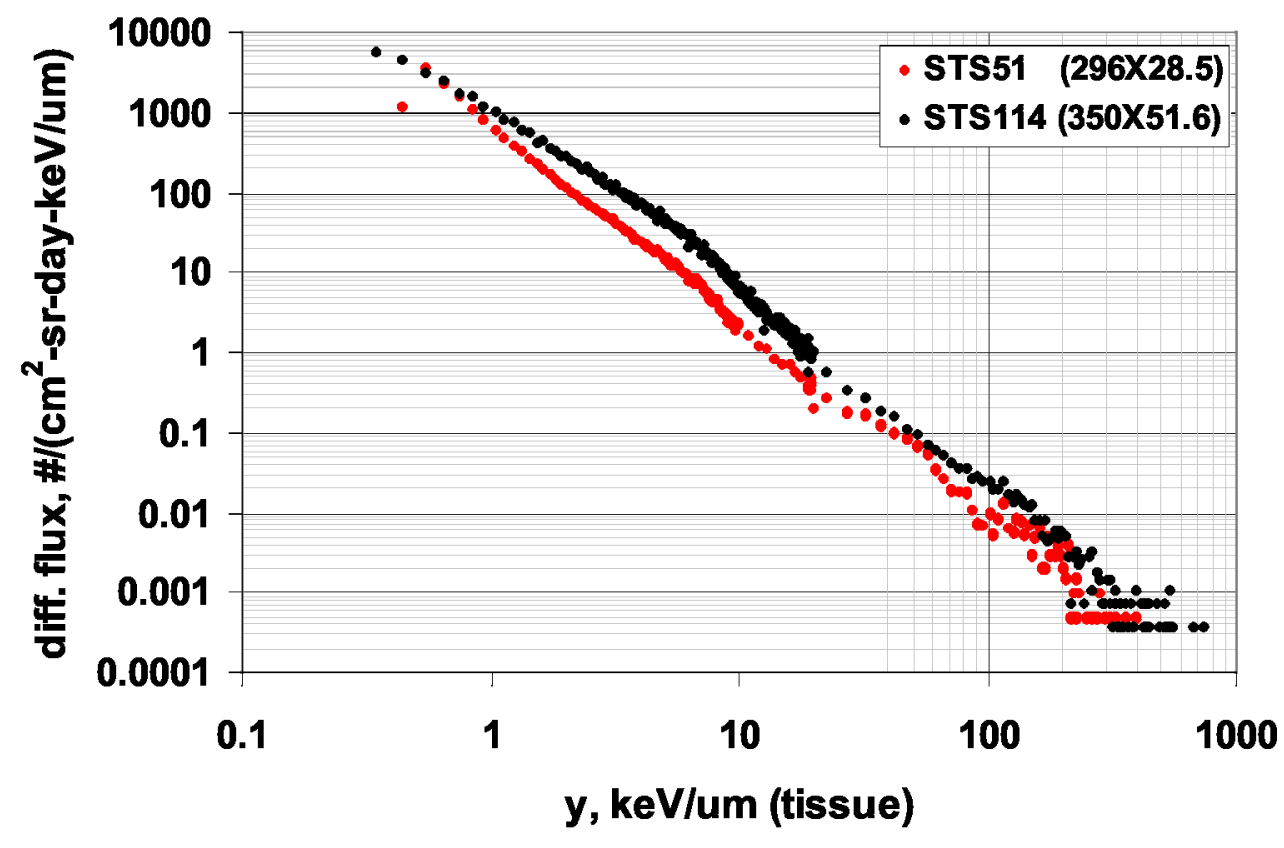

Figure 7a. Measured TEPC GCR differential y spectrum.

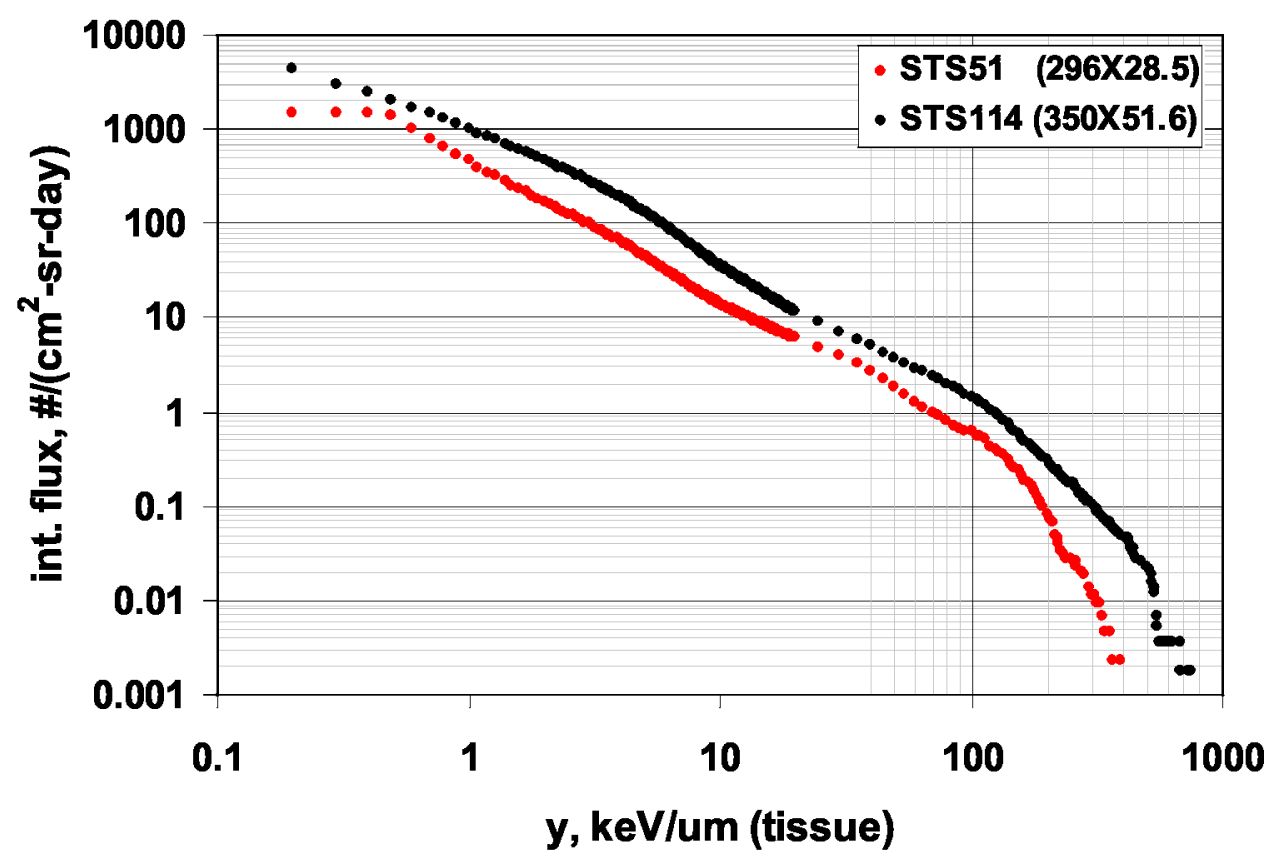

Figure 7b. Measured TEPC GCR integral y spectrum. 
Figures $8 \mathrm{a}$ and $8 \mathrm{~b}$ show the calculated GCR differential response function in y, LET and TEPC measured spectra for STS 51 and 114. For the STS 51 flight, TEPC was mounted at pb\#2, and for the STS 114 flight at dloc2. The proton component of the trapped field in the measurements, being essentially non-existent except for the 10 minutes or so passage through SAA, were eliminated by resolving the time of occurrence for each orbit. The STS shield distribution for pb\#2 and dloc2, as described in figure 5 , were used in the present calculation assuming equivalent aluminum to be the sole shielding material with isotropic incidence of the radiation field on the vehicle. Also, incorporated into the computation are the target fragments contribution with the target material assumed to be tissue (water) representing tissue equivalent plastic wall surrounding the detector gas, which is of sufficient thickness to affect the fragments composition. The presence of spikes in the differential spectrum related to each ion species disappear in the calculated response function (y) for all flights due to the effects of energy loss straggling, smearing from various contributing ions and their energies, and chord length distribution. The predicted y spectrum is seen to slightly improve the agreement with TEPC differential measurements over comparison with the LET spectrum in the region below $10 \mathrm{keV} / \mu \mathrm{m}$. Nevertheless, there is a consistent underestimation in the computed differential results below $10 \mathrm{keV} / \mu \mathrm{m}$ for both flights. This is an indication that there might be problems in the orbit averaged geomagnetic transmission function which is affected by the changes in the Earth's magnetic field. Note that the gradually increasing high noise level above $100 \mathrm{keV} / \mu \mathrm{m}$ in the differential measurement is due to low count (sampling) rate and the channel resolution $(5 \mathrm{keV} / \mu \mathrm{m})$ of the TEPC instrument.

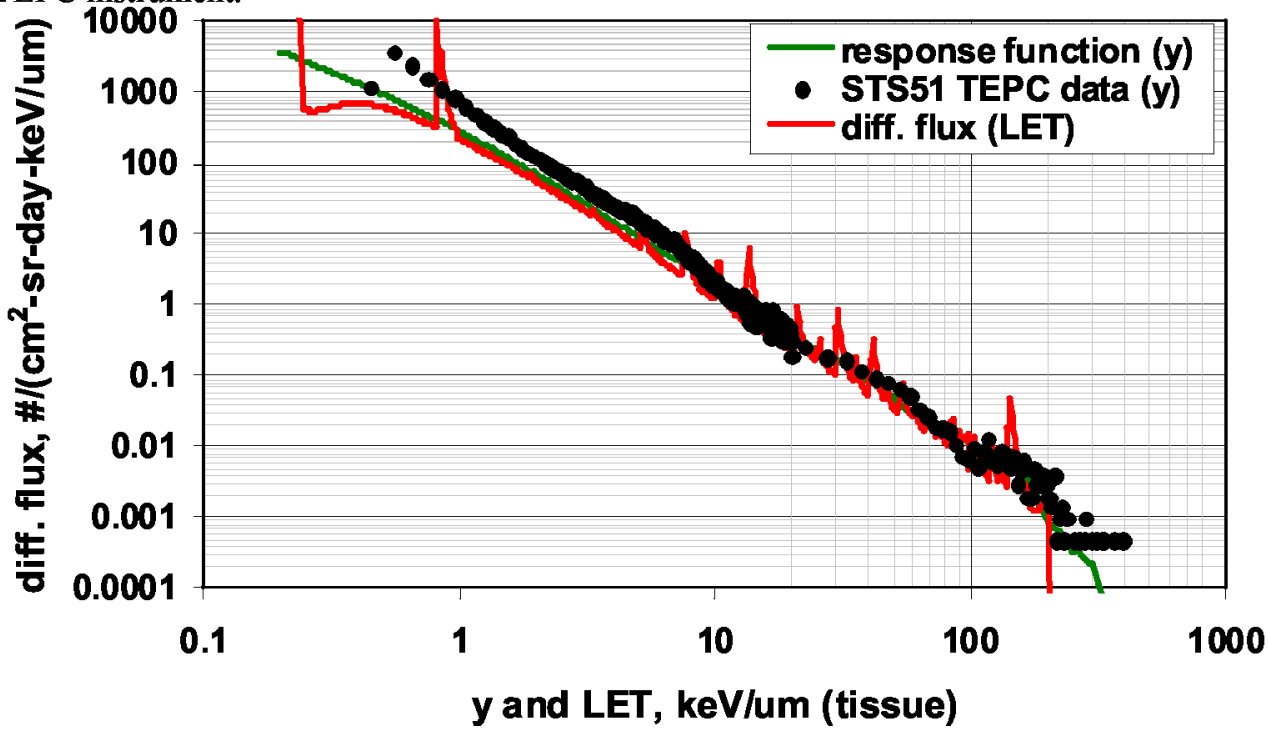

Figure 8a. Comparison of differential y, LET and TEPC Measurement for STS 51.

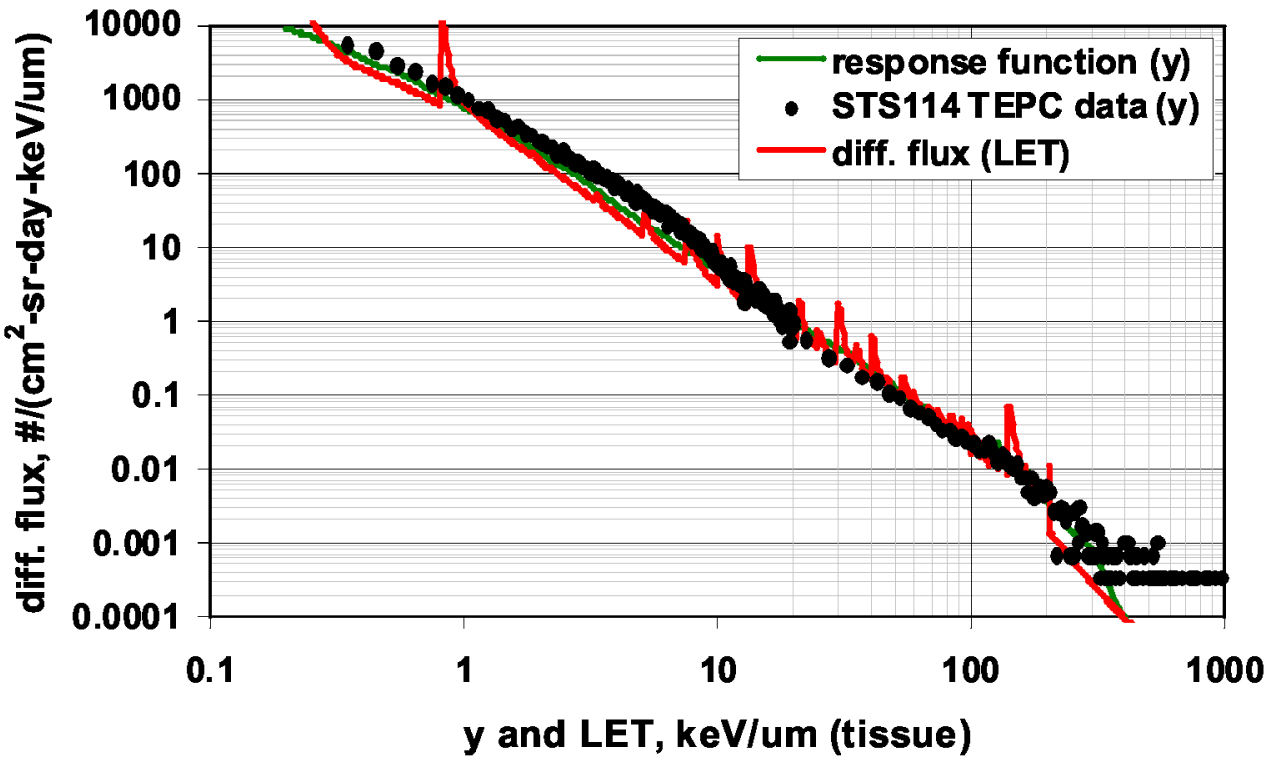

Figure 8b. Comparison of differential y, LET and TEPC Measurement for STS 114. 
Figures $9 \mathrm{a}$ and $9 \mathrm{~b}$ are the corresponding calculated GCR integral response function in $\mathrm{y}$, LET and TEPC measured spectrum for the two STS flights. The calculated integral y spectrum is seen to smooth out the edges related to the minimum ionization peak of alpha and iron ions. In producing the integral $y$, a limiting factor of setting the computed results to zero for $\mathrm{y}>400 \mathrm{keV} / \mu \mathrm{m}$ was implemented to mimic the loss of measured data beyond this $\mathrm{y}$ value. Otherwise, there would have been significant contributions to the integral spectrum above $400 \mathrm{keV} / \mu \mathrm{m}$. The same underestimation below $10 \mathrm{keV} / \mu \mathrm{m}$ in the computed results, as was previously discussed, is also seen in figures $9 \mathrm{a}$ and $9 \mathrm{~b}$.

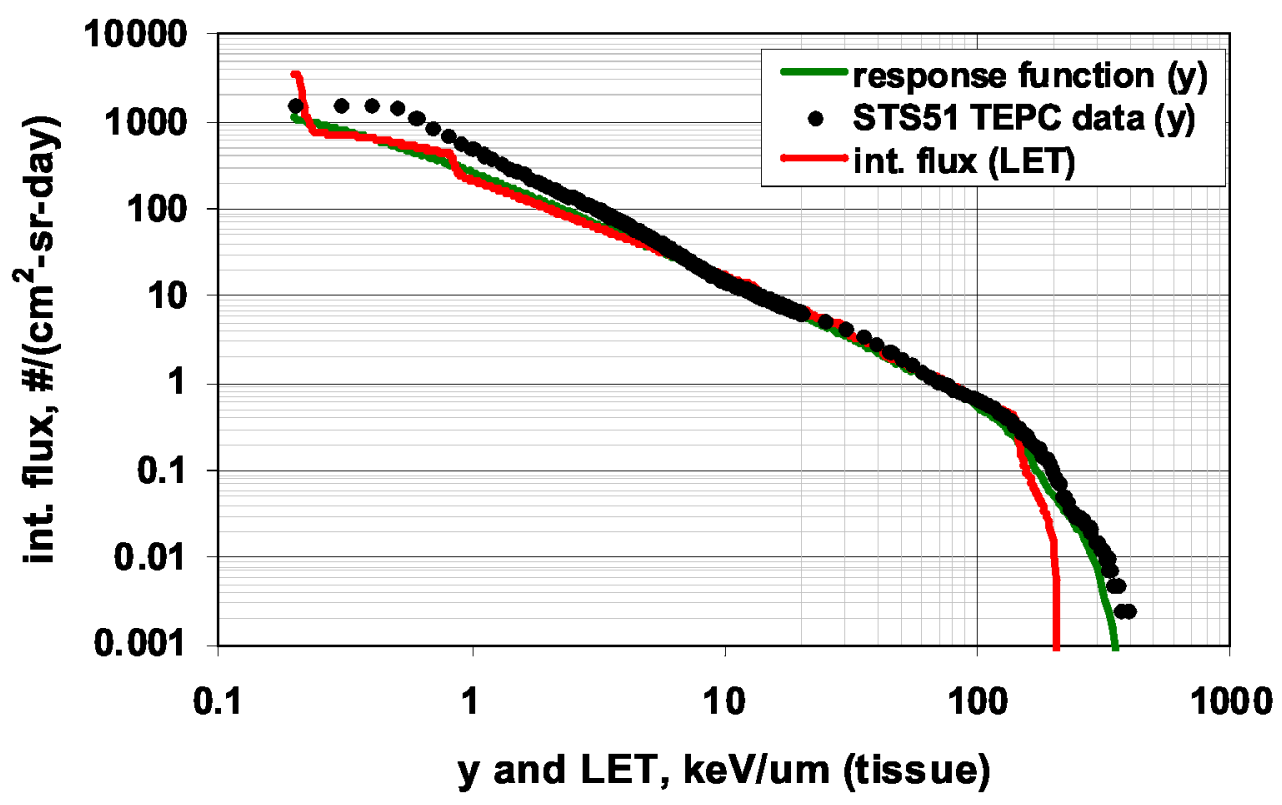

Figure 9a. Comparison of integral y, LET and TEPC Measurement for STS 51.

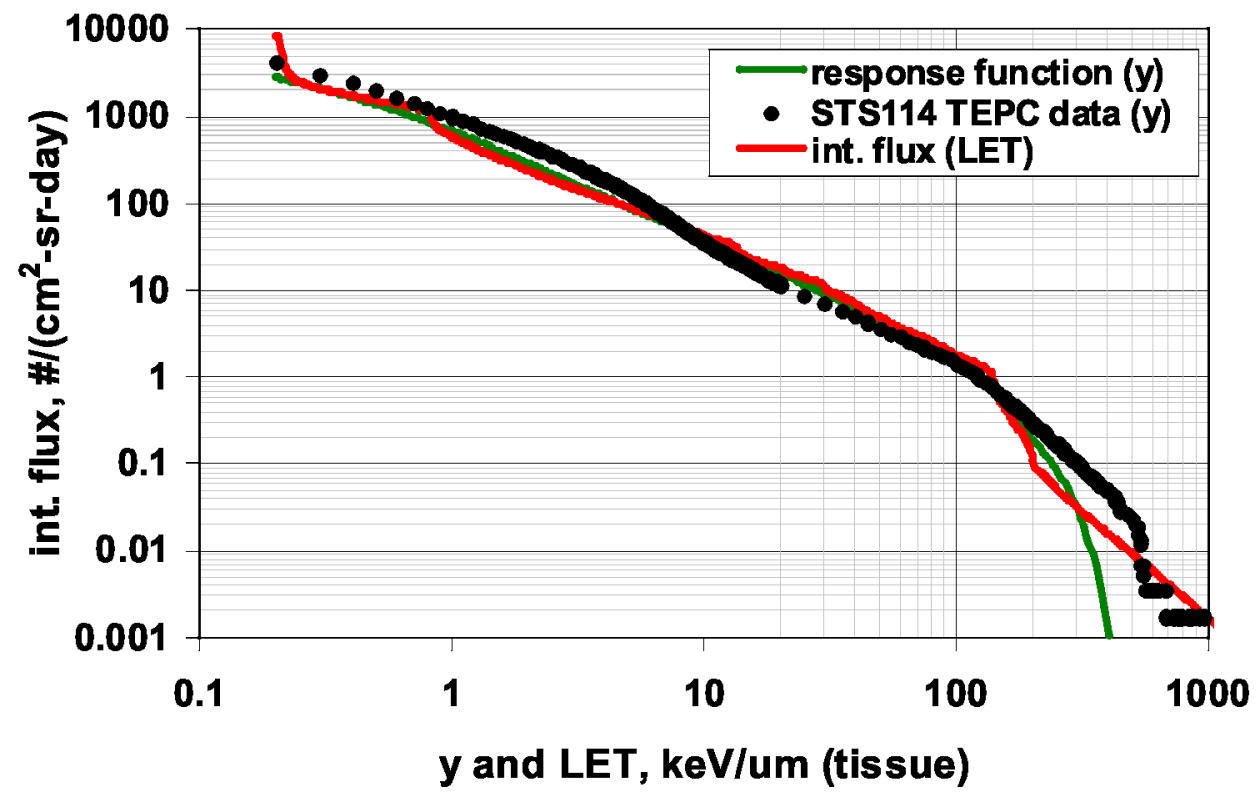

Figure 9b. Comparison of integral y, LET and TEPC Measurement for STS 114. 


\subsection{Trapped Protons Results}

Figures $10 \mathrm{a}$ and $10 \mathrm{~b}$ are the measured trapped protons differential and integral y spectra for the two STS flights. The number of events detected by the TEPC instrument was converted to flux according to the description provided in section 4.1. Note that the proton readouts by the instrument occur only during the 5 to 10 minutes SAA transit per orbit as indicated by the presence of the spikes in figure 3. Both figures indicate that at lower altitude (STS51), the instrument readout is considerably less, as SAA proton flux density is strongly altitude dependent.

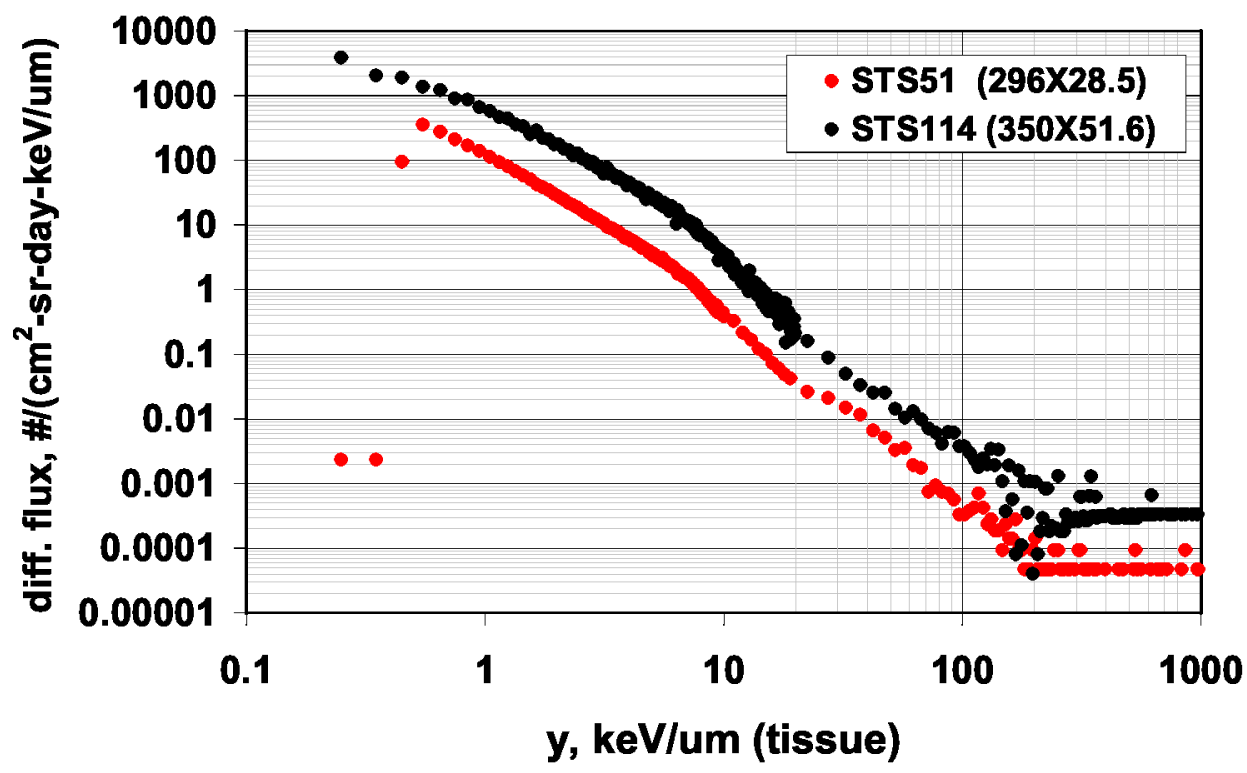

Figure 10a. Measured TEPC trapped protons differential y spectrum.

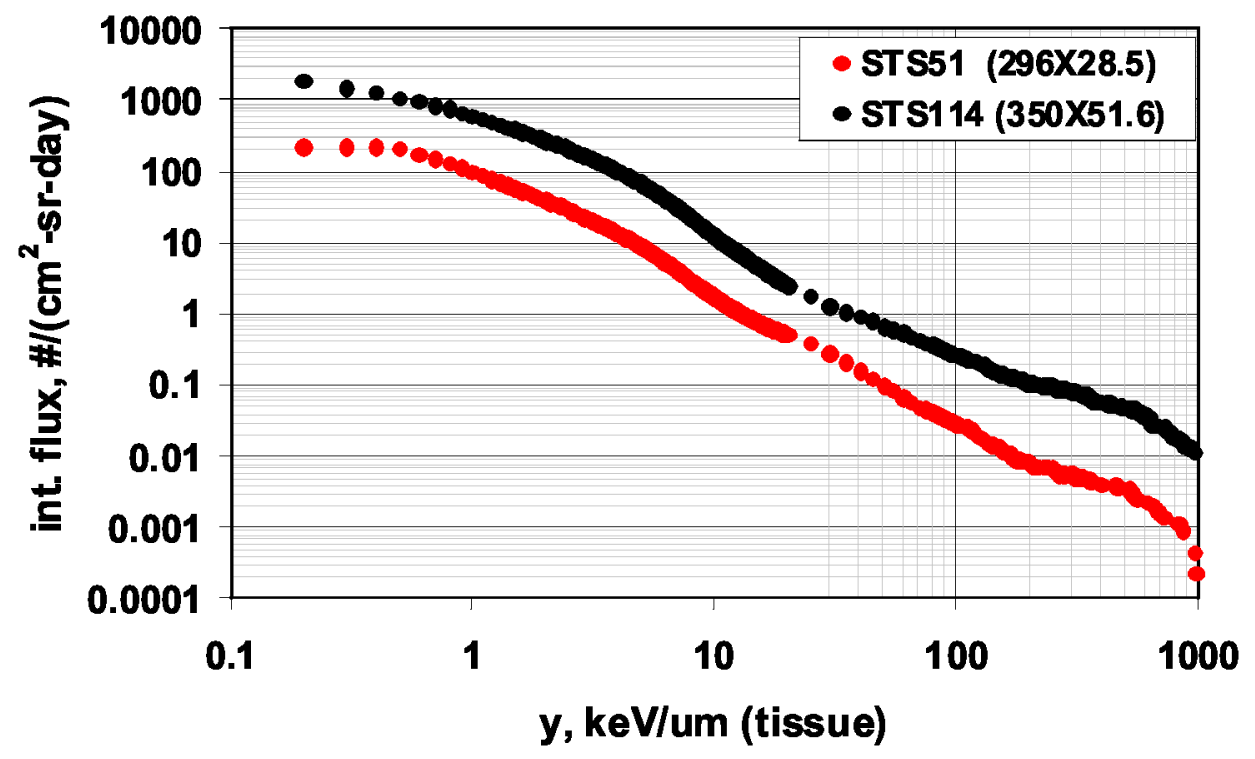

Figure 10b. Measured TEPC trapped protons integral y spectrum.

Figures 11a and $11 \mathrm{~b}$ show the calculated trapped protons differential response function in y, LET and TEPC measured spectrum for STS 51 and 114. For the STS 51 flight, the TEPC was mounted at the pb\#2, and for the STS 114 flight at the dloc2. The GCR components of the measurement were eliminated by resolving the time of occurrence for each orbit according to the time history of the flight (see figure 3). The STS shield distribution for pb\#2 and dloc2, as described in figure 5, were used in the present calculation, assuming equivalent aluminum to be the sole shielding material with isotropic incidence of the radiation field on the vehicle. Also incorporated into the computation are the target fragments contribution with the target material, assumed to be tissue (water) representing tissue equivalent plastic wall surrounding the detector gas, which is of 
sufficient thickness to affect the fragments composition. The presence of two spikes in the differential LET spectrum related to proton

and alpha ions species disappear in the calculated response function (y) for all flights due to the effects of energy loss straggling, smearing from various contributing ions and their energies, and chord length distribution. The predicted y spectrum is seen to slightly improve the agreement with TEPC differential measurements over comparison with the LET spectrum in the region below $10 \mathrm{keV} / \mu \mathrm{m}$. Nevertheless, there is a consistent overestimation in the computed differential results below 10 $\mathrm{keV} / \mathrm{\mu m}$ for both flights. This overestimation is less pronounced for STS 51 as compared with STS 114. Note also that the gradually increasing high noise level above $100 \mathrm{keV} / \mu \mathrm{m}$ in the differential measurement is due to low count (sampling) rate and the channel resolution $(5 \mathrm{keV} / \mu \mathrm{m})$ of the TEPC instrument.

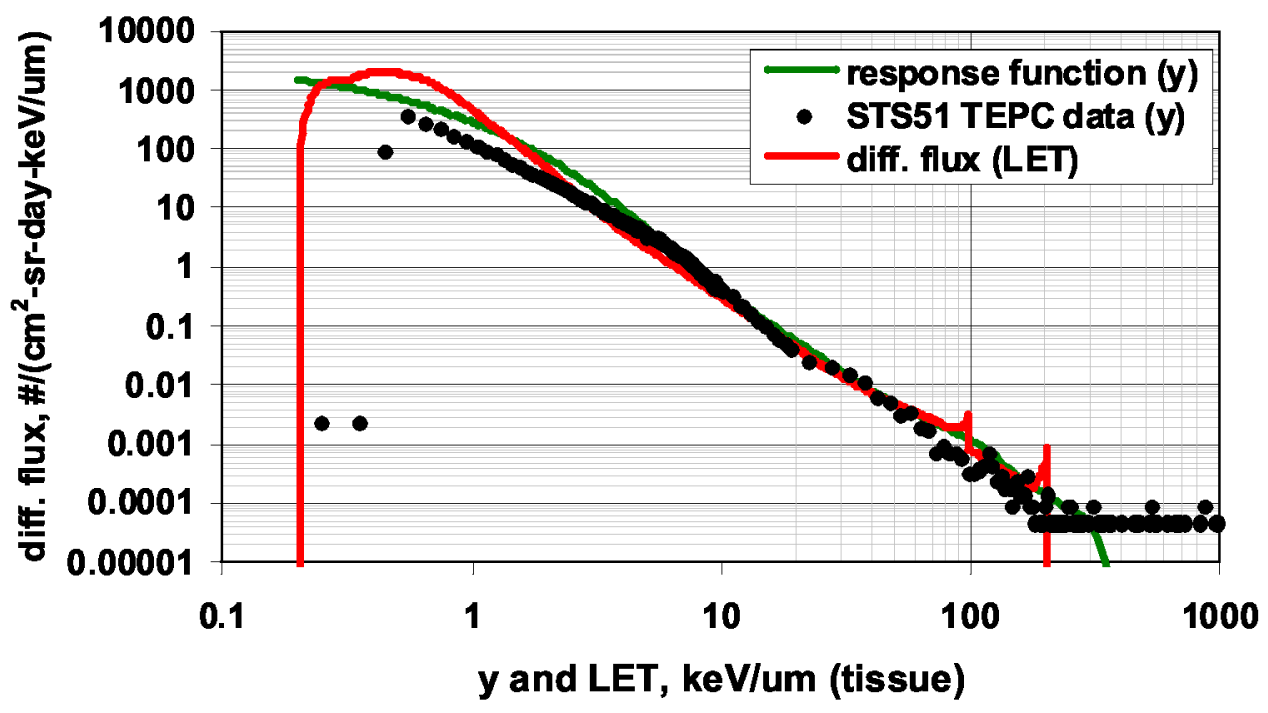

Figure 11a. Comparison of differential y, LET and TEPC Measurement for STS 51.

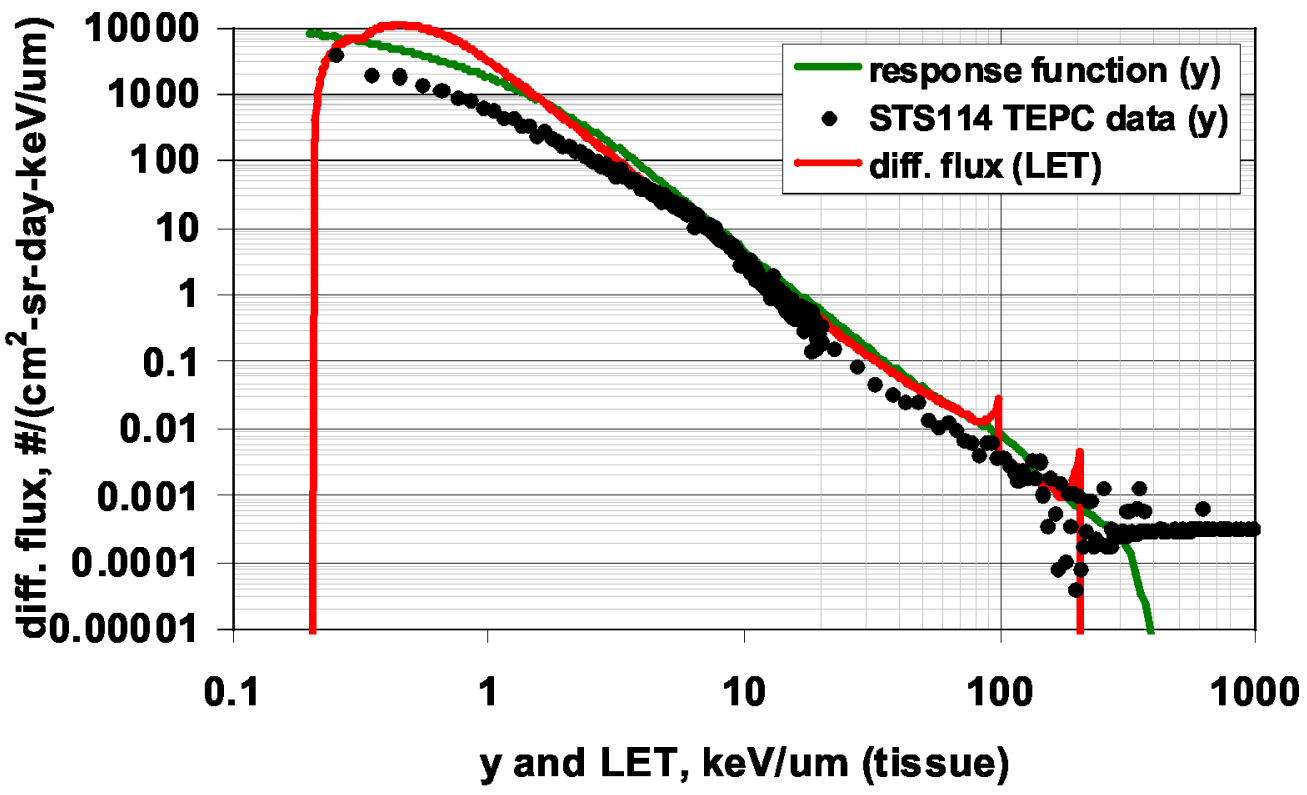

Figure 11b. Comparison of differential y, LET and TEPC Measurement for STS 114.

Figures $12 \mathrm{a}$ and $12 \mathrm{~b}$ are the corresponding calculated trapped protons integral response function in $\mathrm{y}$, LET and TEPC measured spectrum for both STS flights. The calculated integral y spectrum is seen to smooth out the edges related to the minimum ionization peak of proton and alpha ions. The same overestimation, due to the use of an idealized trajectory, below $10 \mathrm{keV} / \mu \mathrm{m}$, in the computed results, is also seen in figures $12 \mathrm{a}$ and $12 \mathrm{~b}$. 


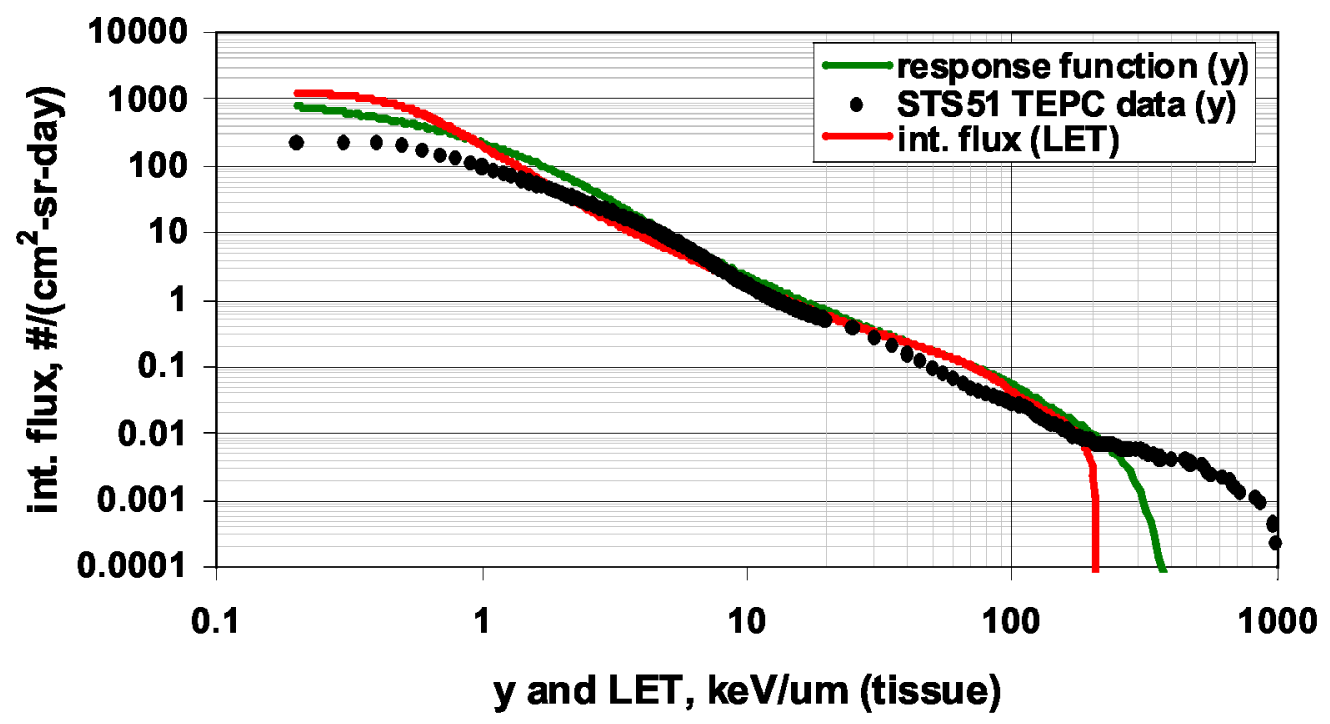

Figure 12a. Comparison of integral y, LET and TEPC Measurement for STS 51.

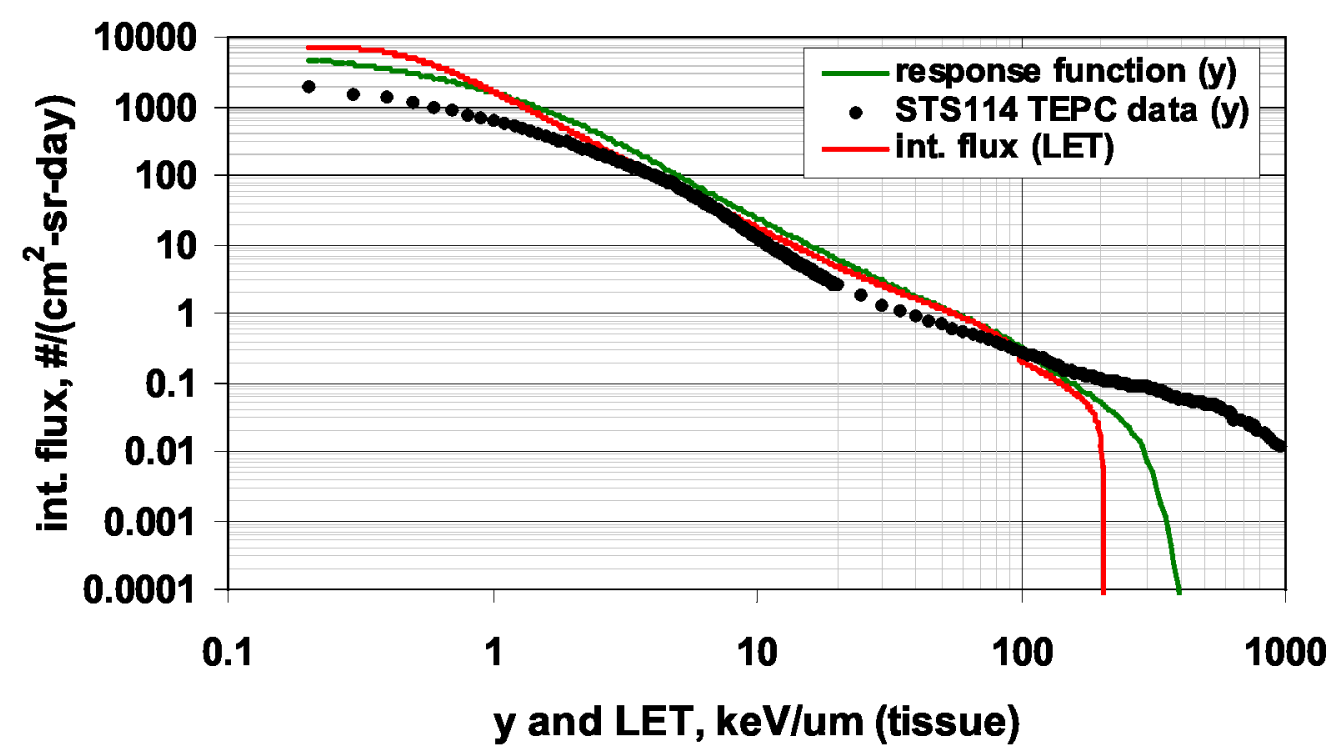

Figure 12b. Comparison of integral y, LET and TEPC Measurement for STS 114.

\section{Conclusions}

GCR and trapped protons differential and integral y and LET spectra were calculated and compared with STS flights 51 and 114 at an orbital inclination range of $28.5^{\circ}-51.6^{\circ}$. The ion transport calculations were made using the latest version of HZETRN (2005). The calculation of energy deposition in the micron size TEPC detector was accomplished using a generalized analytical model which considered both direct and indirect events. The correlation between the computed differential and integral y and LET spectra and TEPC measurements are promising. For the GCR, there seems to be a uniform underestimation of $\mathrm{y}$ and LET spectra below $10 \mathrm{keV} / \mu \mathrm{m}$ as compared with TEPC measurement. This underestimation trend is consistent in both STS flights but with varying magnitude in flux level, suggesting possible problems with the geomagnetic transmission function, which traditionally has used vertical cut-off only. Added to the underestimation is also the exclusion of secondary pions and electrons in HZETRN. However, the significance of these secondary particles can only be analyzed after these secondary interactions are incorporated into HZETRN. For the trapped protons during SAA transits, there is a consistent overestimation of y and LET spectra below $10 \mathrm{keV} / \mu \mathrm{m}$, as compared with TEPC measurements. This overestimation is less pronounced for STS 51 as compared with STS 114. Future improvements of the geomagnetic cutoff model, and addition of secondary particle production in HZETRN will result in a more accurate TEPC correlation with the response function model at low to mid y range. 


\section{References}

1. Kellerer, A. M., Chmelevsky, D., Criteria for the applicability of LET, Radiat. Res., 63, 226-234, 1975.

2. Benton, E. V., Collver, M. M., Registration of heavy ions during the flight of Gemini VI, Health Phys., 13, 495-500, 1967.

3. Badhwar, G. D., Cucinotta, F. A., Braby, L. A., Konradi, A., Measurements on the shuttle of the LET spectra of galactic cosmic radiation and comparison with the radiation transport model, Radiat. Res., 139, 344-351, 1994.

4. Olko, P., Booz, J., Energy deposition by protons and alpha particles in spherical sites of nanometer to micrometer diameter, Radiat. Environ. Biophys., 28, 1-17, 1990.

5. Wilson, W. E., Paretzke, H. G., A stochastic model of ion track structure, Radiat. Protect. Dosim., 52, 249-253, 1994.

6. Xapsos, M. A., Burke, E. A., Shapiro, P., Summers, G. P., Energy deposition and ionization fluctuations induced by ions in small sites: an analytical approach, Radiat. Res., 137, 152-161, 1994.

7. Xapsos, M. A., Burke, E. A., Shapiro, P., Summers, G. P., Probability distributions of energy deposition and ionization in sub-micrometer sites for condensed media, Radiat. Meas., 26, 1-9, 1996.

8. Wilson, J. W., Townsend, L. W., Schimmerling, W., Khandelwal, G. S., Khan, F., Nealy, J. E., Cucinotta, F. A., Simonsen, L. C., Shinn, J. L., Norbury, J. W., Transport methods for space radiations, NASA-RP 1257, 1991.

9. Wilson, J. W., Badavi, F. F., Cucinotta, F. A., Shinn, J. L., Badhwar, G. D., Silberberg, R., Tsao, C. H., Townsend, L. W., Tripathi, R. K., HZETRN: Description of a free-space transport and shielding program, NASA-TP-3495, 1995.

10. Wilson, J. W., Tripathi, R. K., Badavi, F. F., Cucinotta, F. A., Standardized radiation shield design method: 2005 HZETRN, ICES 2006, SAE, 2006-01-2109, July 2006.

11. Badhwar, J.D., O’Neill, P. M., An improved model of GCR for space exploration mission, Nucl. Tracks Radiat. Meas., 20, 403-410, 1992.

12. Nymmik, R. A., Panasyuk, M. L., Pervaja, T. I., Suslov, A. A., A model of galactic cosmic ray fluxes, Nucl. Tracks Radiat. Meas., 20, 427-429, 1992.

13. Chen, J., Chenette, D., Guzik, G., Garcia-Munoz, M., Guzzik, T. G., Pyle, K., Sang, Y., Wefel, J., A model of galactic cosmic rays for use in calculating linear energy transfer spectra, Adv. Space Res., 14, 765-769, 1994.

14. O'Neill, P. M., Badhwar-O'Neill Galactic cosmic ray model update based on advanced composition explorer (ACE) energy spectra from 1997 to present, Adv. Space Res., 37, 1727-1733, 2006.

15. Badavi, F. F., West, K. J., Nealy, J. E., Wilson, J. W., Abrahms, B. L., Luetke, N. J., A dynamic/anisotropic low Earth orbit (LEO) ionizing radiation model, NASA-TP- 214533, 2006.

16. Nealy, J. E., Simonsen, L. C., Qualls, G. D., Cummings, B. C., Sharney, L. J., Modeled environment and exposures for the SAGE-III instrument configuration, ANS Nuclear technologies for Space Exploration, Jackson, WY, Aug. 1619, 1992.

17. Shinn, J. L., Wilson, J. W., Badavi, F. F., Benton, E. V., Csige, I., Frank, A. L., Benton, E. R., HZE beam transport in multilayered materials, Radiat. Meas., 23, 57-64, 1994.

18. Cucinotta, F. A., Townsend, L. W., Wilson, J. W., Shinn, J. L., Badhwar, G. D., Dubey, R. R., Light ion component of the galactic cosmic rays: Nuclear interactions and transport theory, Adv. Space Res., 17, 77-86, 1995.

19. Wilson, J. W., Tripathi, R. K., Cucinotta, F. A., Shinn, J. L., Badavi, F. F., Chun, S. Y., Norbury, J. W., Zeitlin, C. L., Heilbronn, L., Miller, J., NUCFRG2: An evaluation of the semiempirical nuclear fragmentation database, NASATP-3533, 1995. 
20. Kellerer, A. M., Microdosimetry and the theory of straggling, In Biophysics Aspects of Radiation Quality, $2^{\text {nd }}$ Panel Report, International Atomic Energy Agency, Vienna, 89-123, 1968.

21. Fano, U., Ionization yields of radiations. II. The fluctuations of the number of ions, Phys. Rev. 72, 26-29, 1947.

22. Aitchison, J., Brown, J. A. C., The lognormal distribution, Cambridge University Press, Cambridge, U. K., 1957.

23. Xapsos, M. A., A spatially restricted linear energy transfer equation, Radiat. Res., 132, 282-287, 1992.

24. Xapsos, M. A., Applicability of LET to single events in microelectronic structures, IEEE Trans. Nucl. Sci., 39, 1613$1621,1992$.

25. Vail, P. J., Burke, E. A., Fundamental limits imposed by gamma dose fluctuations in scaled mos gate insulators, IEEE Trans. Nucl. Sci., 31, 1411-1416, 1984. 